\title{
Research on the Application of VPython 3D Dynamic Visualization in the Teaching of College Physics
}

\author{
Tao Wu ${ }^{1 *}$, Qian Wang1, Shifang Wang2 \\ ${ }^{1}$ School of Optical Information and Energy Engineering, Wuhan Institute of Technology, Wuhan 430205, China \\ ${ }^{2}$ School of Physics and Electromechanical Engineering, Hubei Second Normal University, Wuhan 430205, China \\ *Corresponding author: Tao Wu, wutao@wit.edu.cn
}

\begin{abstract}
As there are numerous physics formulas in addition to them being theoretical, college students generally consider the concepts of physics as abstract, in which they are not able to apply what they have learned. For 3D dynamic animation and visualization in the teaching of college physics, VPyton has many advantages, and it provides useful exploration for students to overcome obstacles in college physics learning. Taking the teaching of simple harmonic vibration, optics, molecular thermal motion, and electric field as examples, this article discusses the application of VPython in the humancomputer interactive teaching mode of college physics, which provides a beneficial exploration and new way to improve students' engineering skills and numeracy, as well as to respond to the national "new engineering education" reform in China.
\end{abstract}

Keywords: College physics; VPython; Animation and visualization teaching; New engineering

Publication date: September 2021; Online publication: September 30, 2021

\section{Introduction}

As an important general education course in science and engineering schools, college physics plays an important role in the cultivation of college students' scientific quality. The development of the internet and information technology have greatly changed the traditional teaching mode, resulting in various teaching modes, such as presentation-assimilation-discussion (PAD) class ${ }^{[1]}$, flipped class ${ }^{[2]}$, and blended class ${ }^{[3]}$. In addition, mobile network learning and online teaching have also become the widely used methods for college students to acquire knowledge. As a basic course, college physics has been at the forefront of the teaching mode reform in this new era, accumulating a large number of online teaching resources and creating a batch of online demonstration courses like massive open online courses (MOOCs) and golden courses ${ }^{[4,5]}$. Modern physics presents a three-pronged pattern of theoretical physics, experimental physics, and computational physics. The popularization and development of computers have also greatly promoted the application of graphical and visual teaching methods in college physics. In the internet age, the importance of computer language is unquestionable, in which it is an essential skill for every undergraduate student.

In learning college physics, the biggest challenge for students is that the concepts of physics are abstract and difficult to understand. There are many theoretical derivations and formulas with unclear physical pictures; thus, they are unable to apply what they have learned. Under the background of promoting the construction of "new engineering" and "double first-class," the language of scientific computing is deeply integrated into the theoretical and practical teaching of college physics courses, which can play an important enlightening and promoting role in cultivating students' engineering skills and thinking. In order to solve 
these unresolved problems and respond to the national "new engineering" professional construction call, this article proposes a teaching reform measure for the 3D dynamic visualization of basic physics by making full use of VPython, a free, open-source software, and third-party libraries based on the Python language. This can fundamentally reduce the students' fear of learning college physics, increase their interests in learning, and lay a certain foundation for cultivating innovative talents in the new era.

\section{Analysis on the advantages of VPython 3D dynamic visualization in the teaching of college physics}

The Python language is one of the most popular languages at present. It is simple and easy to learn. In recent years, a large number of free, open-source software and scientific computing extension libraries based on the Python language have been developed, such as NumPy, SciPy, SymPy, Pandas, and Matplotlib, which provide Python with fast array processing, symbolic calculation, numerical calculation, and drawing functions. Therefore, the development environment formed by the Python language and its many extended libraries is extremely suitable for engineering and scientific researchers to process experimental data, make charts, and even develop scientific computing applications, especially interactive teaching platforms based on the Jupyter Lab or Notebook, which can seamlessly import a large number of rich third-party libraries to achieve 3D visualization in the teaching of college physics as exemplified by VPython which can create 3D scenes and animations as well as built-in a large number of functions and classes to achieve rapid programming of college physics teaching content and 3D visualization display physical pictures.

In regard to the interactive and visualized teaching of college physics, commercial software such as MATLAB, Mathematica, and Origin can be replaced by the Python language as well as its rich, fast-updated, and constantly evolving third-party libraries. In the learning process of theoretical physics knowledge, it acts as the carrier and provides practical application scenarios for students to train their numeracy and engineering skills. In addition, it provides a good platform for students to combine theory with practice and solve practical problems. The advantages of Vpython in 3D dynamic visualization for the teaching of college physics are mainly seen in a few aspects.

(1) Python language has developed rapidly due to its powerful computing ecology. The rich Pythonbased third-party software library is fast in updates and has strong technical scalability and community maintenance, which can be integrated on the Jupyter interactive teaching platform. It provides a web-based version of GlowScript and can also build a server teaching platform. Its popularity, generality, and extensibility provide a variety of selective and sustainable application prospects.

(2) The programming language is simple and clear in order to prevent students from spending a great deal of time in learning language specifications while studying physics. Through this easy-to-learn programming language, students can quickly begin learning to simulate physical process by computer programming and the physical pictures behind the abstract physical formulas.

(3) VPython can realize 3D animation to simulate physical process, which is convenient for displaying physical phenomena in a 3D space. It provides an opportunity to choose the observation angle for a learning experience of human-computer interaction mode and improving students' interest in learning.

(4) The best way for learning is to learn by doing. In the teaching process, students are guided to use VPython language for programming practice to realize college physics graphics. 


\section{Examples of 3D visualization of college physics teaching with VPython}

\subsubsection{D dynamic visualization of simple harmonic vibration}

In the teaching of harmonic vibration, first of all, a teacher can use VPython's rich built-in 3D objects to construct a 3D teaching scene of the horizontal spring vibrator. Through the Python code, the 3D dynamic movement of the harmonic vibration particles can be freely controlled, allowing students to intuitively and vividly obtain simple harmonic motion pictures. In addition to the analytical method for solving the simple harmonic vibration differential equation, it can also be solved numerically based on the numerical solution program of Runge-Kutta or Euler method based on Python, and the vibration curve of the simple harmonic vibration can then be drawn. In addition to the analytical method, the dynamic rotation vector method can be used for the description of the simple harmonic vibration. Based on the VPython programming, the rotation vector method of the simple harmonic vibration can be animated, and the synthesis of the simple harmonic vibration can be studied based on the rotation vector method.
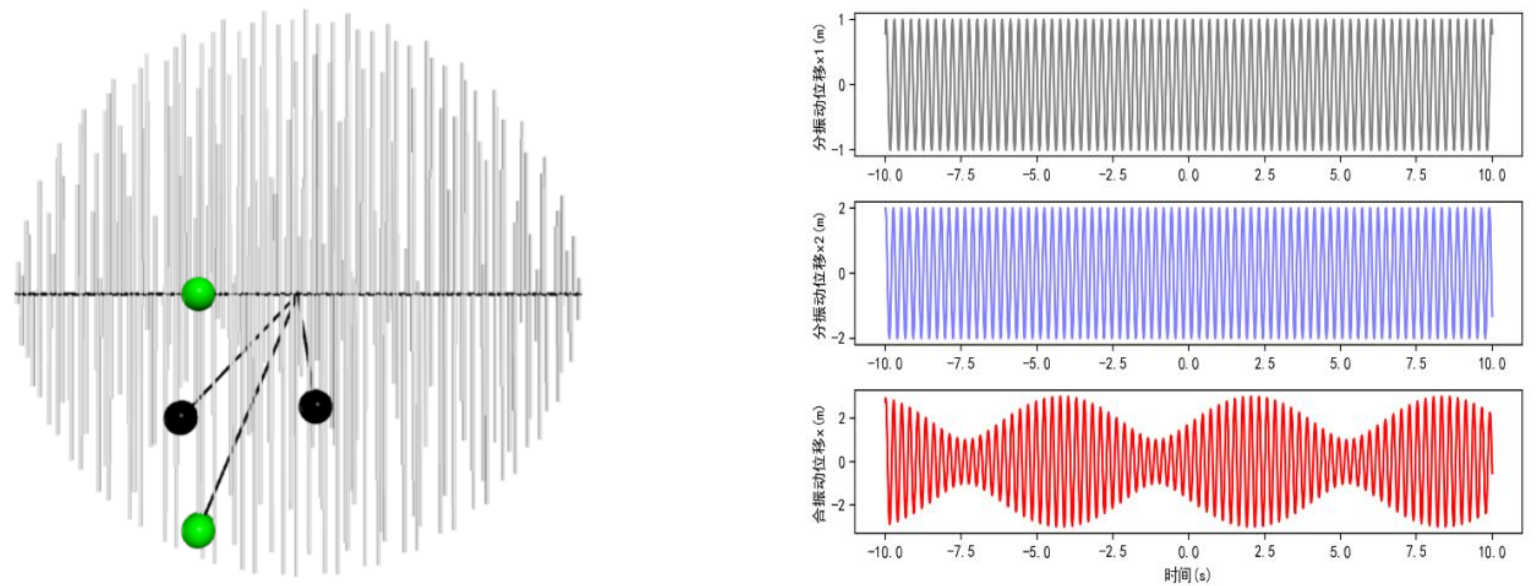

Figure 1. 3D animation visualization screenshot based on VPython simple harmonic vibration beat formation

Figure 1 shows the 3D animation visualization screenshot based on VPython simple harmonic motion synthesis, and simultaneously demonstrates the vibration curves of the sub-vibration and the composite vibration. The animation demonstration of VPython does not only provide the opportunity for students to obtain physical pictures visually, but also to modify the program code by themselves, realize interactive learning, improve students' interest in learning, and provide training opportunities for students to solve practical problems and improve their programming skills with the knowledge they have learned.

\subsection{Optical 3D visualization}

In the teaching of physical optics in colleges, VPython can be used to quickly construct teaching scenes, such as light refraction, reflection, thin lens, spherical mirror imaging, and light polarization. Programming calculations based on the Huygens-Fresnel principle can also restore 3D animation scenes, such as light interference and diffraction. Figure 2 shows a screenshot of the 3D visualization of reflection, refraction, and circular aperture diffraction of light based on VPython. These 3D dynamic scenes can intuitively display the main principles and simulate experimental phenomena in the learning of physical optics. In addition, they are able to display the laws and scenes of physics, thus helping students to quickly learn and master relevant theoretical knowledge in physics. 

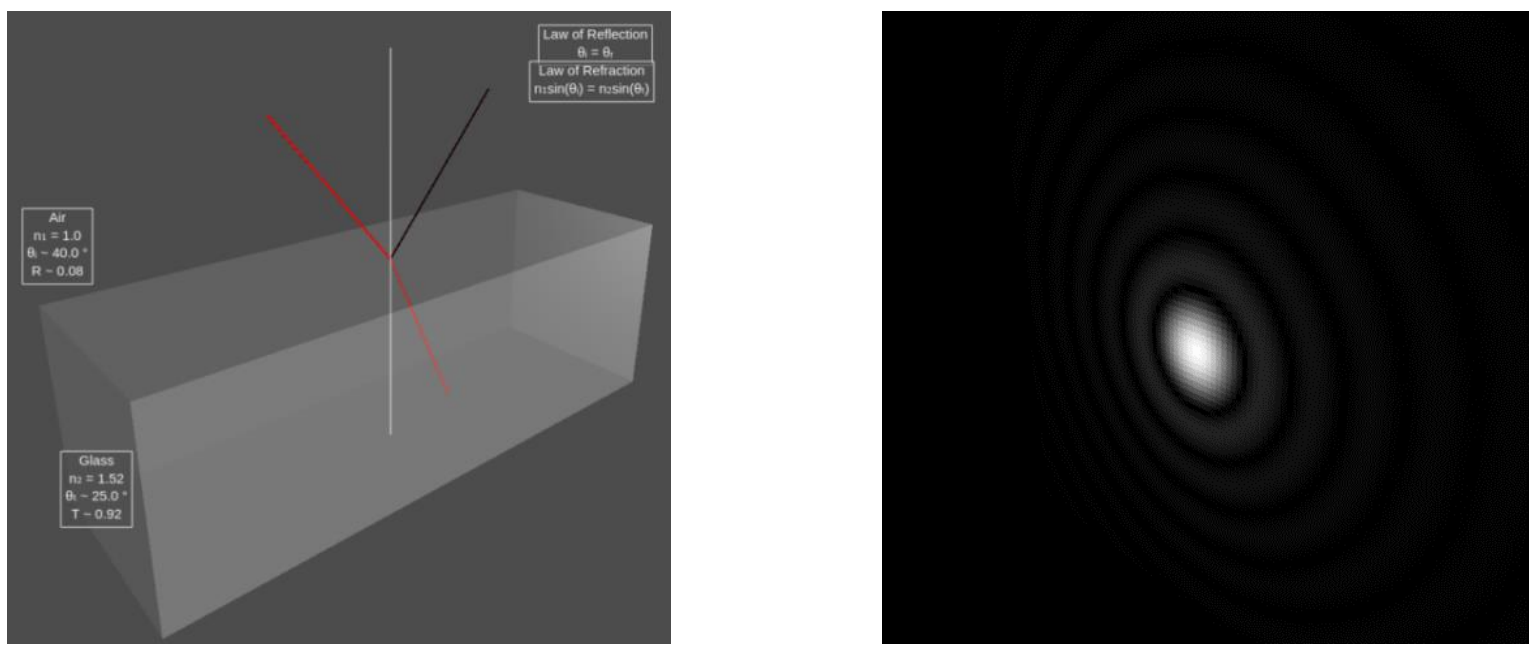

Figure 2. 3D visualization of light reflection, refraction, and circular aperture diffraction based on VPython

\subsection{D dynamic visualization of molecular thermal motion}

In the teaching of molecular thermal motion, the physical pictures of an ideal gas and molecular thermal motion occurs in 3D space. The microscopic particles continue to collide, and their motion state changes with time, which is very complicated, but statistical regularity appears in thermal equilibrium. These abstract physics concepts are the key to college physics courses, but the visualization and imaging of these processes are not easy to obtain from experiments. For students to grasp these concepts, teachers can use VPython to simulate the physical process in order to restore physical pictures and reinforce students' understanding and mastery.
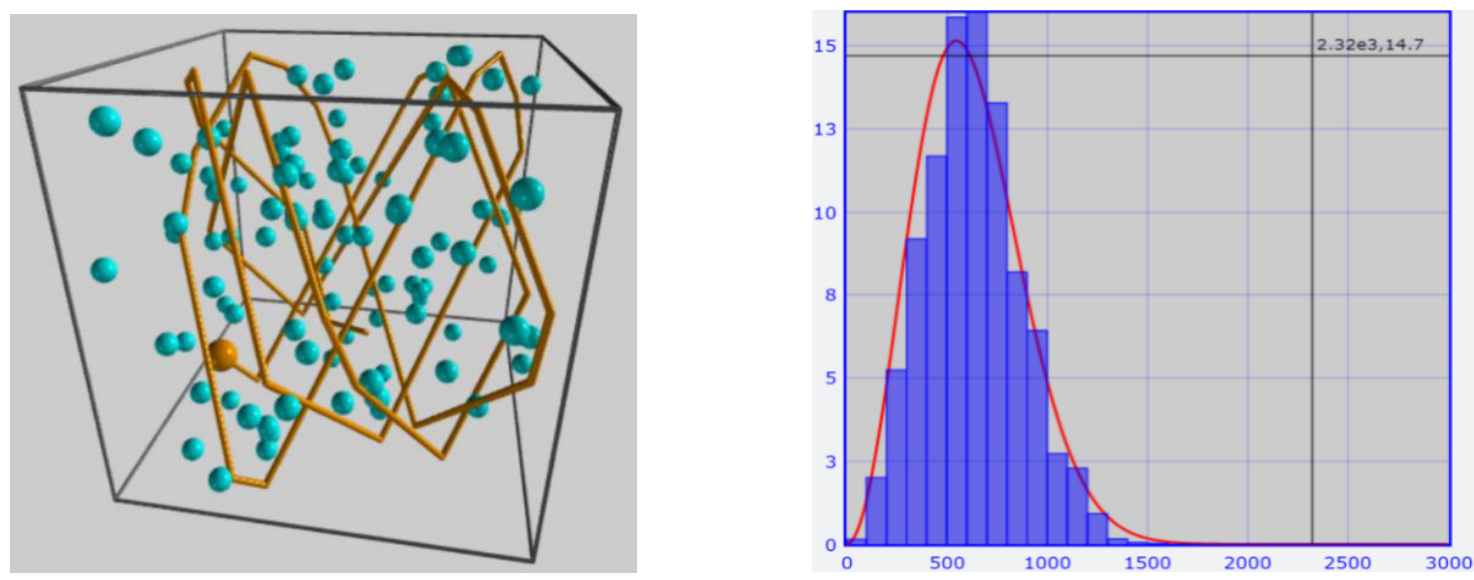

Figure 3. Screenshot of the 3D visualization animation of molecular thermal motion and Maxwell's distribution law

Figure 3 shows a screenshot of the 3D visual simulation animation of VPython molecular thermal motion and Maxwell's distribution law. This figure also shows the thermal motion trajectory of a specific gas molecule that is represented by a yellow ball. As time goes by, the histogram on the right in Figure 3 shows that the rate of movement of molecules is closer to Maxwell's distribution law, which is in agreement with the theoretical curve in red and tends to be stable.

\subsection{D visualization of electric field lines}

In the study of electric and magnetic fields, the concept of electromagnetic fields in 3D space is abstract 
and difficult to understand. In addition, the distribution of electric and magnetic fields in different situations are very complicated. It is quite difficult to obtain the distribution of these fields through experiments. VPython provides a point charge sphere object, where people can set the charge amount it carries. By programming based on Coulomb's law, the electric field distribution excited by different point charge systems can be calculated and an electric field line diagram can be obtained. Figure 4 shows the 3D model diagram of electric dipole as well as the electric field distribution and electric field line diagrams through VPython. For the picture on the left, students can choose the space field point to obtain the electric field vector of the point interactively, while the picture on the right shows the electric field diagram of the electric dipole.
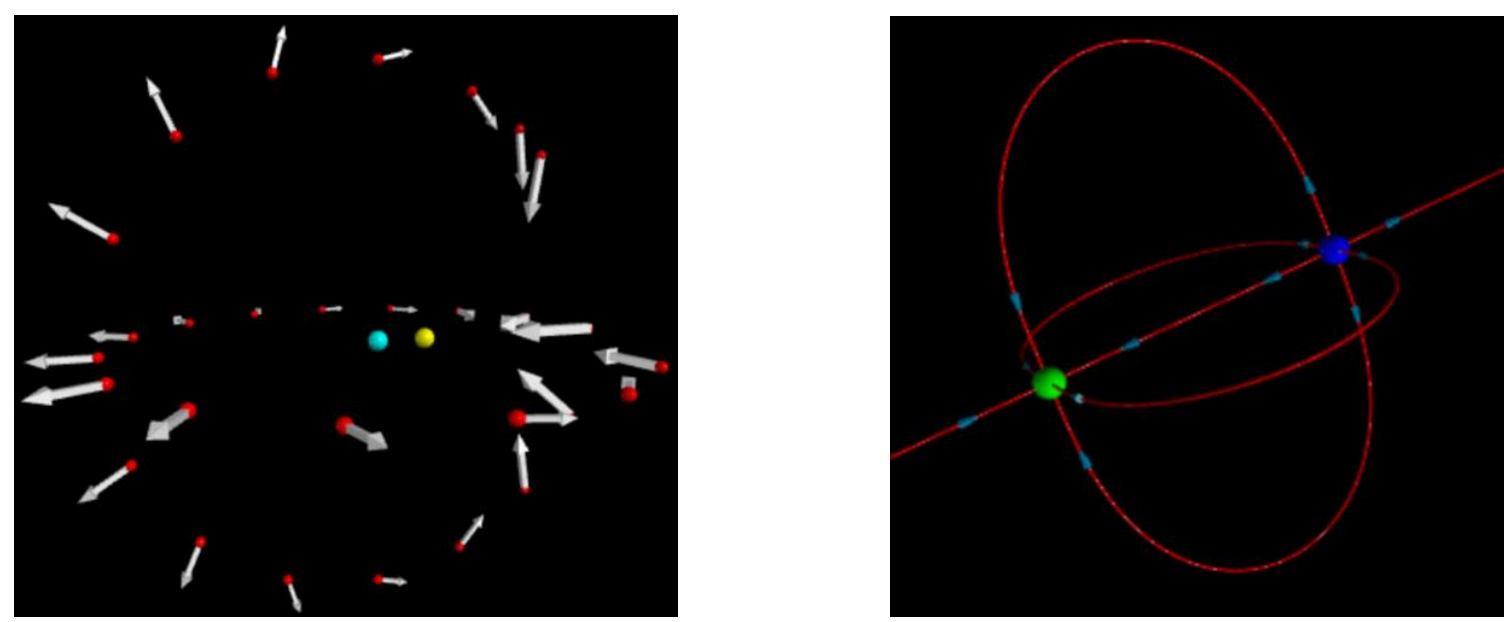

Figure 4. 3D visualization of electric dipole, electric field distribution, and electric field lines by VPython

These images can be rotated and moved arbitrarily in three dimensions, which is convenient for students to observe the field distribution from different angles. VPython also provides a linear model object for current-excited magnetic fields, where students can set the current that passes through them and program based on Biot-Savart law to calculate the magnetic field distribution as well as plot the magnetic field. After classes, students can be assigned exercises in regard to VPython programming calculations for electric and magnetic field distributions stimulated by various charge and current distributions. This would encourage students to learn physics concepts, master programming skills, as well as cultivate numeracy and engineering skills by doing these exercises on their own.

\section{Conclusion}

VPython, as a new 3D visualized teaching simulation library, is not only suitable for the teaching of college physics, but also meets the teaching needs of different disciplines and has a wide range of applications for interactive teaching in foreign universities. In China, the application of VPython for interactive teaching started a little later than in foreign countries, and its application examples are lacking. Many physical process simulations still need to be developed. The actual operation is not matured enough, and there is still a lot of room for development. From the perspective of pilot teaching and practice, students can apply their acquired skills in other subjects or physics problems after they have been trained in VPython programming simulation in order to create fresh simulation results of physical phenomena. In the process of learning, although students need to invest more energy to complete after-class programming assignments, the results would be very fulfilling, and they would be able to achieve a deeper understanding of the physical picture and meaning behind the physical formulas. The adoption of this teaching model can greatly stimulate 
students' learning potential and provide useful exploration as well as new ways to improve students' engineering skills, numeracy, and respond to the talent training mode of the national "new engineering education" reform in China.

\section{Funding}

This work is funded by the Wuhan Institute of Technology project titled, "The Reform and Practical Teaching of the New Engineering Education Visualization Teaching Mode of College Physics Based on Jupyter Lab/Notebook Platform" and a top course of the online undergraduate courses of college physics in Hubei Province as well as Guangdong Major Project of Basic and Applied Basic Research (No. 2019B030302003).

\section{Disclosure statement}

The author declares that there is no conflict of interest.

\section{References}

[1] Qin D, Xing J, Li G, etc., 2021, A Practical Exploration of the Teaching Mode of PAD Class in College Physics. College Physics, 40(4): 32-36.

[2] Hao D, Zhang L, Chen S,2020, College Physics Classroom Research under the Flipped Classroom Teaching Mode. Science and Technology Vision, (34): 48-49.

[3] Xu F, Luo D, Xie H, et al., 2021, Research on Flipped Classroom Teaching Mode of College Physics Experiment Based on MOOC Platform. Physics and Engineering, 31(1): 50-54.

[4] Wang H, Wu P, Zhu H, etc., 2021, Practice of Online and Offline Blending-Learning Mode of College Physics Based on the Construction of Golden Course. College Physics, 40(3): 51-55, 61. 